\title{
ELOITA PEREIRA NEVES: BALUARTE DA ENFERMAGEM DA UNIVERSIDADE FEDERAL DE SANTA CATARINA
}

\author{
Miriam Süsskind Borenstein ${ }^{1}$, Maria Emília de Oliveira², Evanguelia Kotzias Atherino dos Santos ${ }^{3}$, \\ Isabel Cristina Alves Maliska ${ }^{4}$
}

\footnotetext{
${ }^{1}$ Doutora em Filosofia da Enfermagem. Professor Associado do Departamento de Enfermagem e do Programa de Pós Graduação (PEN) da Universidade Federal de Santa Catarina (UFSC). Pesquisadora do CNPq. Santa Catarina, Brasil. E-mail: miriam@ nfr.ufsc.br

${ }^{2}$ Doutora em Enfermagem. Professor Associado do Departamento de Enfermagem da UFSC. Santa Catarina, Brasil. E-mail: mila@nfr.ufsc.br

${ }^{3}$ Doutora em Filosofia da Enfermagem. Professor Associado do Departamento de Enfermagem e do PEN/UFSC. Santa Catarina, Brasil. E-mail: gregos@matrix.com.br

${ }^{5}$ Doutoranda do PEN/UFSC. Enfermeira do Hospital Universitário da UFSC. Santa Catarina, Brasil. E- mail: isabel.alves07@ yahoo.com.br
}

\begin{abstract}
RESUMO: Trata-se de um estudo sócio-histórico cujo objetivo foi historicizar a trajetória profissional de Eloita Pereira Neves, apresentar alguns traços de sua biografia e analisar sua contribuição para a Enfermagem da Universidade Federal de Santa Catarina. Na coleta de dados foram consultadas fontes documentais e complementares. Os dados foram organizados de acordo com a análise de conteúdo. Os resultados evidenciam que a Professora Eloita teve participação expressiva no serviço de saúde hospitalar local, na Associação Brasileira de Enfermagem - Seção Santa Catarina, inclusive ocupando cargos nesta última, que culminaram entre outros, na criação do primeiro Curso de Graduação em Enfermagem em Santa Catarina. Sua formação e participação ativa contribuíram para que mudanças ocorressem na enfermagem catarinense a partir da criação do primeiro Curso de Graduação em Enfermagem da Universidade Federal de Santa Catarina, que hoje tem reconhecida atuação no ensino, pesquisa e extensão no âmbito estadual, nacional e internacional.
\end{abstract}

DESCRITORES: Educação. Biografia. Educação em enfermagem. História da enfermagem.

\section{ELOITA PEREIRA NEVES: NURSING'S REFERENCE AT THE FEDERAL UNIVERSITY OF SANTA CATARINA}

\begin{abstract}
This is a socio-historical study aimed to historicize the career of Eloita Pereira Neves, present some features of her biography, and analyze her contribution to nursing at the Federal University of Santa Catarina, Brazil. Data were collected through consulting documentary and complementary sources. The data were organized according to content analysis. The results showed that Professor Eloita had significant participation in the health service hospital site and the Brazilian Nursing Association - Section Santa Catarina, including holding key positions in the latter, which resulted among others in creating the first undergraduate Nursing course in Santa Catarina. Her professional education and active participation contributed so that significant changes occurred in nursing in the state of Santa Catarina, creating the first undergraduate course at the Federal University of Santa Catarina, now recognized for its teaching, research, and outreach on state, national, and international levels.
\end{abstract}

DESCRIPTORES: Education. Biography. Nursing education. Nursing History.

\section{ELOITA PEREIRA NEVES: BALUARTE DE LA ENFERMERÍA DE LA UNIVERSIDADE FEDERAL DE SANTA CATARINA}

\begin{abstract}
RESUMEN: Se trata de un estudio socio-histórico cuyo objetivo fue historiar la trayectoria profesional de Eloita Pereira Neves, presentar algunos trazos de su biografía y analizar su contribución para la Enfermería en la Universidade Federal de Santa Catarina. Fueron consultadas fuentes documentales y complementarias. Los datos fueron organizados de acuerdo con Bardin. Los resultados evidenciaron que la Profesora Eloita tuvo participación expresiva en el servicio de salud hospitalaria local, en la Asociación Brasilera de Enfermería - Sección Santa Catarina, inclusive ocupando cargos en esta última, que culminaron entre otros, en la creación del primer Curso de Graduación en Enfermería en Santa Catarina. Su formación y participación activa contribuyó para la enfermería catarinense a partir de la creación del primer Curso de Graduación en Enfermería de la Universidade Federal de Santa Catarina, que hoy tiene reconocida actuación en la enseñanza, investigación y extensión en el ámbito estadual, nacional e internacional.
\end{abstract}

DESCRIPTORES: Educación. Biografía. Educación en Enfermería. Historia de la Enfermería 


\section{INTRODUZINDO A TEMÁTICA}

Os primeiros cursos de enfermagem que surgiram no Brasil foram criados durante a Primeira República, segundo a proposta de modelos europeus, sendo que dentre esses, podemos destacar: a atual Escola de Enfermagem Alfredo Pinto, da Universidade Federal do Estado do Rio de Janeiro (UNIRIO) e os Cursos da Cruz Vermelha. ${ }^{1}$ Posteriormente, em 1923, foi criada a Escola de Enfermeiras do Departamento Nacional de Saúde Pública (DNSP) por iniciativa do eminente sanitarista e cientista Carlos Chagas (Diretor do recém criado DNSP) e graças aos esforços das enfermeiras americanas que integraram a Missão Técnica de Cooperação para o desenvolvimento da Enfermagem no Brasil. Esta escola surgiu no contexto do Movimento Sanitário Brasileiro do início do século XX e, foi fundamental para se constituir como modelo às demais escolas que passaram a ser criadas no país. Com o Decreto No 17.268 de 31 de março de 1926, passou a chamar-se Escola de Enfermeiras D. Ana Néri, implantando a carreira de enfermagem - modelo "Nightingale" em nível nacional. ${ }^{2}$ Em 1937, esta escola foi incorporada à Universidade do Brasil e passou a chamar-se Escola Anna Nery, caracterizando-se como um marco histórico da enfermagem brasileira ao tornar-se a Escola de Enfermagem padrão. A partir de sua criação, outras escolas passaram a ser criadas em todo o território nacional sendo que, ao final dos anos 60, já havia no país trinta e dois cursos de enfermagem em funcionamento, dos quais vinte oito $(87,5 \%)$ eram governamentais ou mantidos por Congregações religiosas, e apenas quatro (12,5\%) eram vinculados à estabelecimentos com fins lucrativos. ${ }^{1}$

Em Santa Catarina, o primeiro Curso de Graduação em Enfermagem foi criado em 24 de janeiro de 1969, através da Resolução No 02/69, do Magnífico Reitor João David Ferreira Lima, agregado a Faculdade de Medicina da Universidade Federal de Santa Catarina (UFSC), em Florianópolis. Essa foi uma grande conquista para os profissionais que atuavam na capital e interior, pois até então, o Estado carecia de escolas de formação em enfermagem. Os poucos enfermeiros que aqui residiam e trabalhavam eram provenientes de outros estados, em especial do Rio Grande do Sul, Paraná e São Paulo. ${ }^{3}$

A criação do Curso de Graduação em Enfermagem na capital, trouxe significativas trans- formações nos serviços de saúde e melhoria na assistência de enfermagem à população, devido à inserção dos egressos do curso nas instituições de saúde do estado. Com o passar dos anos, o corpo docente do curso começou a preocupar-se com a qualificação de seus profissionais sendo que, em 1976 foi criado o Programa de Pós-graduação em Enfermagem (PEN) Strictu Sensu, o Mestrado, e em 1992, o Doutorado. Atualmente o Departamento de Enfermagem e o PEN têm forte inserção regional, destacando-se no cenário nacional e internacional. Até o presente momento, o Curso de Graduação formou 1.808 enfermeiros e o PEN 494 mestres e 163 doutores."

Ao comemorarmos os 40 anos de criação do Curso e Departamento de Enfermagem da UFSC em 2009, não poderíamos deixar de homenagear uma das professoras que mais contribuiu para que esse Curso/Departamento chegasse a ser o que é atualmente. Reconhecida nacional e internacionalmente, a Prof ${ }^{a}$. Dra. Eloita Pereira Neves foi a principal responsável e articuladora pelo planejamento, criação e implementação deste curso na década de 60 . Foi a primeira coordenadora do Curso de Graduação, Chefe do Departamento e professora, e teve um papel crucial no desenvolvimento do mesmo.

Considerando a importância e contribuição que a professora teve a frente do Departamento de Enfermagem e do PEN/UFSC, o presente estudo teve como objetivo historicizar a trajetória profissional de Eloita Pereira Neves, apresentar alguns traços de sua biografia e analisar sua contribuição para a Enfermagem na UFSC.

Acreditamos que através desse estudo historiográfico, possamos resgatar a trajetória de uma personalidade de grande expressão no contexto da enfermagem, e ao mesmo tempo, prestarmos uma homenagem justa e de valor a quem tanto contribuiu para a profissão no cenário catarinense e nacional.

\section{METODOLOGIA}

Trata-se de um estudo sócio-histórico, cujos dados foram obtidos a partir de consulta a livros, capítulos de livros, artigos de periódicos, currículo lattes da biografada, teses e outras fontes documentais. Os dados foram analisados utilizando-se do método de análise de conteúdo, ${ }^{4}$ sendo estabelecidas as seguintes categorias de análise: Formação;

Dados obtidos pelas autoras no Departamento de Enfermagem e no Programa de Pós-graduação em Enfermagem em 20 de outubro de 2009. 
A participação na Associação Brasileira de Enfermagem (ABEn) - Seção SC; O envolvimento da Professora Eloita Pereira Neves com a criação do Curso de Graduação em Enfermagem junto a UFSC; O Caminho trilhado na pós-graduação e O reconhecimento pela trajetória acadêmica e profissional.

\section{APRESENTAÇÃO DOS RESULTADOS}

\section{Formação}

Eloita Pereira Neves nasceu em 17 de maio de 1940, na cidade de Lages, Santa Catarina (SC), é filha de Celso Vieira Neves e Senhorinha Pereira Neves. Realizou seus estudos (primário, secundário, normal e contabilidade) em sua cidade natal. Ao concluir o normal, passou a lecionar na Escola Nossa Senhora do Rosário em Lages-SC, onde permaneceu pelo período de um ano.

Apesar de já ser professora (o que era considerado como uma profissão muito boa para as mulheres, à época - anos 60), sentiu a necessidade de continuar seus estudos, optando por ingressar em um curso superior. Desde a infância demonstrava o desejo de trabalhar em uma maternidade, como consequência, a enfermagem seria uma excelente opção. Em 1960, resolveu prestar o vestibular para a Escola de Enfermagem de Porto Alegre (EEPA) da Universidade Federal do Rio do Grande do Sul. A EEPA além de possuir um bom ensino, oferecia moradia, alimentação e uniforme.

Nas provas do vestibular, Eloita se classificou em $5^{\circ}$ lugar, entre as vinte e quatro candidatas. Enquanto aluna do curso de graduação, além de se dedicar com afinco aos estudos, demonstrava também engajamento político ativo no Centro Acadêmico, participando em 1962, do Congresso da União Nacional de Estudantes na cidade de Petrópolis-RJ e também do Movimento Estudantil que reinvindicava a Reforma Universitária. Após três anos de estudos, diplomou-se em 8 de dezembro de 1963 , tendo obtido o $2^{\circ}$ lugar da turma. ${ }^{5}$ Em 1968 , bacharelou-se em Educação pela Universidade para o Desenvolvimento do Estado de Santa Catarina.

Com a conclusão do Curso de Graduação em Enfermagem, inúmeras oportunidades de emprego surgiram em Porto Alegre-RS, entretanto Eloita, por ser catarinense e sentindo-se comprometida em contribuir com seu Estado, retornou ao mesmo, atendendo ao convite formulado pelo Dr. Miguel Salles Cavalcanti, para trabalhar no Hospital Infantil Edith Gama Ramos (HIEGR). Este médico seria o futuro diretor geral dessa instituição, recém cons- truída em Florianópolis-SC. Assim, juntamente com outra colega da EEPA, a também catarinense Irmgard Bruckheimer e três outras enfermeiras recém tituladas (Nelcy Terezinha Pacheco Coutinho, Leonor Caon e Nilza Borges Paim) pela Faculdade de Enfermagem Madre Justina Inês de Caxias do Sul-RS, iniciou suas atividades profissionais no HIEGR. Estas enfermeiras, juntamente com Eloita, chegaram à Capital em janeiro de 1964, passando imediatamente a atuar no HIEGR, que tinha muito para ser feito, pois havia sido recém inaugurado. Organizaram o Serviço de Enfermagem, reestruturaram a área física, fizeram solicitação de materiais e equipamentos necessários, elaboraram o organograma, o regimento interno, e os manuais de enfermagem, entre outros. ${ }^{5}$

\section{A participação na Associação Brasileira de Enfermagem-Seção SC}

A cidade de Florianópolis, que até então carecia de enfermeiras, recebeu um grupo competente e responsável que além de integrar o Serviço de Enfermagem do HIEGR, passou também a atuar ativamente na ABEn-SC.

Esta Seção, criada em 13 de março de 1962, por influência direta da Irmã Cacilda (Ottillie Hammes), Diretora da Escola de Auxiliares Madre Benvenuta, foi uma grande conquista das enfermeiras catarinenses. Isto devido ao fato do estado contar com um grupo reduzido de profissionais, pois as que aqui residiam, estavam vinculadas com a ABEn do Rio Grande do Sul, que pela distância geográfica e até mesmo cultural, não satisfaziam as necessidades das catarinenses. ${ }^{6}$

A ABEn-SC teve um papel importantíssimo, agregando ao seu quadro todas as profissionais que aqui chegavam, entre elas a enfermeira Eloita, que durante sua trajetória profissional assumiu inúmeros cargos nesta Associação. Foi secretária em 1964; presidente na gestão 1966-1967; tesoureira em 1970, na gestão de Nelcy Terezinha Coutinho Mendes; e coordenadora da comissão de educação no ano de 1972, sob a presidência da enfermeira Ingrid Elsen.?

As enfermeiras costumavam reunir-se frequentemente para deliberar acerca de assuntos diversos relativos a profissão. Apesar da associação ter sido criada essencialmente com a finalidade cultural, de desenvolvimento da profissão, as integrantes envolviam-se em várias atividades, tais como: esclarecimento acerca da profissão de Enfermagem (através dos meios de comunicação), organização de eventos (como por exemplo a Semana da Enfer- 
magem) e até mesmo, fiscalização de cursos (como o de Enfermagem Obstétrica, que vinha sendo ministrado para as parteiras na Maternidade Carlos Corrêa) desde $1935 .{ }^{3,8}$ Este curso, embora fosse para parteiras, era ministrado em sua maioria por um grupo de médicos que atuavam na Maternidade, e não possuía nenhuma enfermeira à frente, somente uma única parteira. ${ }^{9}$ Por esse motivo, as enfermeiras que atuavam na ABEn-SC sentiam-se na obrigação de fiscalizar, uma vez que na época não havia um órgão fiscalizador, sendo o Conselho Regional de Enfermagem criado posteriormente através da Portaria COFEn 01 de 04/08/1975. Por muitos anos, a ABEn-SC respondeu pela representação do grupo profissional, mapeando os enfermeiros em número, local de atuação e atividades que desenvolviam. Em 1964, o grupo pressionou e conseguiu fechar o Curso de parteiras pelo não cumprimento da lei, uma vez que não havia enfermeiras como professoras e supervisoras. A enfermeira Eloita, junto com as demais associadas, teve um papel fundamental no fechamento deste. ${ }^{8}$

A ABEn-SC, através de suas associadas, desenvolvia ainda atividades junto aos órgãos públicos estaduais, discutindo questões relativas à contratação e demissão de pessoal, bem como em relação à criação de um quadro de profissionais de enfermagem para os serviços de saúde. Constituiu bancas para concursos para seleção de enfermeiros em hospitais e para o Departamento Autônomo de Saúde Pública (DASP) e promoveu inúmeros cursos de atualização e treinamentos de profissionais da saúde. $^{8}$

Uma das questões constantemente debatidas pelas associadas, era a necessidade da criação de uma Escola de Graduação em Enfermagem, pois no estado de SC não havia nenhuma. Em março de 1965, durante a 17ª Reunião da ABEn-SC, este assunto foi amplamente discutido, ficando decidido que deveria ser realizado um debate sobre esta questão durante a Semana de Enfermagem, que aconteceria em maio do corrente ano. Entretanto, por conta da crise que passava o país naquele momento (Revolução de 64), o assunto só foi tema de discussão em setembro desse mesmo ano, por ocasião da $25^{\text {a }}$ Assembléia Geral da ABEn-SC. ${ }^{3}$

\section{O envolvimento da Professora Eloita Pereira Neves com a criação do Curso de Graduação em Enfermagem junto à UFSC}

Eloita Pereira Neves, então Presidente da ABEn-SC, resolveu aceitar o desafio de criação da escola, juntamente com as demais associadas, elaborando um documento no qual descrevia a situação da Enfermagem em Florianópolis e a necessidade de um curso que se responsabilizasse pela formação destas profissionais. Pleiteavam junto a UFSC a criação da Faculdade de Enfermagem. Com o apoio de dois pediatras (Nelson Grisard e Gabriel Faraco) que também atuavam no HIEGR, conseguiram articular uma reunião com o Reitor da UFSC à época, o Professor João David Ferreira Lima. ${ }^{3}$

Esta reunião foi a primeira de muitas que aconteceram e, finalmente em novembro de 1966, o Reitor em Exercício, Professor Polydoro Ernani de São Thiago, nomeou através da Portaria $\mathrm{N}^{\circ}$ 364/66, uma Comissão Especial encarregada de realizar os estudos necessários à criação da Escola de Enfermagem. Essa Comissão foi constituída pelos professores Roberto Mündell de Lacerda (Vice-Reitor), Henrique Manoel Prisco Paraíso (Presidente da Associação Catarinense de Medicina) e pela enfermeira Eloita Pereira Neves (Presidente da ABEn-SC). Após vários encontros, a comissão deliberou em reunião realizada em 17 de fevereiro de 1967, sobre a necessidade da ABEn-SC elaborar um estudo para viabilização do curso, estabelecendo os padrões mínimos necessários para o funcionamento do mesmo junto a UFSC. ${ }^{3}$

A ABEn nomeou uma Comissão que foi constituída pelas enfermeiras Eloita Pereira Neves (Presidente da ABEn-SC), Irmã Agnes Edtmann do Hospital Governador Celso Ramos e Irmã Áurea Schmidt, Diretora da Escola de Auxiliar de Enfermagem Madre Benvenuta. Essa comissão, após intenso trabalho, elaborou um relatório informando sobre as condições existentes e as necessárias para o desenvolvimento do curso, incluindo as atividades administrativas e didáticas, campos de atividades práticas e corpo docente. Além de elaborar a proposta, a comissão ocupouse em estudar a legislação que reestruturava as universidades brasileiras, elaborou o regimento e enviou ofícios circulares às instituições, motivando os enfermeiros para participarem do corpo docente e até mesmo da direção do curso. ${ }^{3}$

Para a Direção da Escola houve a indicação de uma professora aposentada da Escola de Enfermagem de Ribeirão Preto, a Profa. Zélia Barbosa Machado. Essa professora veio inúmeras vezes à Florianópolis para participar das reuniões, conhecer os serviços, demonstrando profundo apoio ao Projeto, porém declinou do convite, sugerindo o nome de Eloita, destacando que a mesma além de estar vivamente envolvida com o projeto, caracterizava- 
se como uma enfermeira de grande capacidade e potencial para assumir a direção dessa escola. ${ }^{3}$

Em novembro de 1967, Eloita é contratada como professora titular da UFSC e passa a atuar como coordenadora do futuro Curso de Graduação em Enfermagem, ainda em processo de criação. Com a finalidade de conhecer as questões didáticas, administrativas e financeiras que viria a gerenciar, realizou visitas às principais Escolas de Enfermagem do país (Alfredo Pinto, Luisa de Marillac, Anna Nery, todas localizadas no Rio de Janeiro e às Escolas de Enfermagem da Universidade Federal da Bahia e a do Rio Grande do Sul). No seu retorno, elaborou o Regimento Interno e o Currículo Pleno, de acordo com a Lei No 775/49 e o Parecer N N $^{\circ}$ 271/ 62 do Conselho Federal de Educação e fez contato com as instituições de saúde para atuarem como campo de atividades práticas. ${ }^{3}$

Em julho de 1968, o processo de criação do Curso foi concluído, tendo sido enviado ao Magnífico Reitor David Ferreira Lima, que o encaminhou ao Conselho Universitário para aprovação. O curso foi aprovado neste conselho, por unanimidade, no final de julho do mesmo ano. Desta forma, em 24 de janeiro de 1969, através da Resolução Nº 02/ 69 do Reitor David Ferreira Lima, foi criado o Curso de Graduação em Enfermagem de nível superior, agregado à Faculdade de Medicina da UFSC.

O papel desempenhado por Eloita em todo este processo foi preponderante. Além de participar de toda a discussão para a criação do curso, exerceu as funções de coordenadora e ainda assumiu a responsabilidade no desenvolvimento das disciplinas de Liderança na Assistência de Enfermagem e Administração Aplicada à Enfermagem. Sempre preocupada com a qualidade do ensino, em 1974, sentiu a necessidade de cursar uma Pósgraduação em Enfermagem. ${ }^{5}$

\section{O caminho trilhado na pós-graduação}

O interesse da Professora Eloita em se atualizar, seguindo na carreira universitária estava diretamente vinculado com os novos rumos da Educação Brasileira, que estabelecia a Pós-graduação a partir da década de 1960, quando por solicitação do Ministério da Educação e Cultura, o Conselho Federal de Educação emite um parecer sobre a definição dos Cursos de Pós-graduação. Neste parecer faz-se uma distinção entre a Pósgraduação Lato Sensu e Stricto Sensu. ${ }^{10}$
A Pós-graduação Stricto Sensu em Enfermagem no Brasil teve início em 1972, na Escola de Enfermagem Anna Nery (EEAN) da Universidade Federal do Rio de Janeiro (UFRJ), quando a mesma iniciou o Mestrado em Enfermagem Fundamental. Em 1973, a Escola de Enfermagem da Universidade de São Paulo iniciou o mestrado na área de concentração em Fundamentos de Enfermagem. Em 1975, quatro outras áreas foram criadas: Administração de Serviço de Enfermagem, Enfermagem Psiquiátrica, Enfermagem Pediátrica e Enfermagem Obstétrica. ${ }^{11}$

Eloita, que já vinha acompanhando a política do Departamento em criar um Curso de Pósgraduação em Enfermagem desde 1973, pois este necessitava de uma massa critica docente para ministrar cursos de pós-graduação elevando o nível dos profissionais de enfermagem, ingressou como aluna bolsista da CAPES na EEAN em 1975, a fim de realizar o Mestrado em Enfermagem. Em três anos (1975 a 1977) conseguiu concluir as disciplinas e defendeu a dissertação intitulada "Avaliação da qualidade da assistência de enfermagem - validação de um instrumento", sob a orientação da Professora Maria Dolores Lins de Andrade. ${ }^{12}$

Motivada com os estudos de Pós-graduação que vinha realizando, interessada em participar e implementar o Programa de Pós-graduação em Enfermagem que estava sendo criado na UFSC (1976), estimulada por Cilei Chaves Rhodus, ${ }^{* *}$ e com a obtenção de uma bolsa de doutorado da CAPES, resolve continuar os estudos, indo para os Estados Unidos a fim de cursar o doutorado.

Após a realização de exames seletivos (toefl, entre outros), ingressa novamente como aluna bolsista da CAPES no curso de Doutorado em Ciências da Enfermagem na Catholic University of América, na cidade de Washington, Estados Unidos, permanecendo até 1980, quando concluiu sua tese de doutorado intitulada "The relationship of hospitalized individuals cognitive structure regarding health to their health self care behaviors", tendo como orientadora a Professora doutora Elisa Wolff. ${ }^{12}$

Ao retornar para o Departamento de Enfermagem da UFSC, em 1980, passa a atuar preferencialmente no Programa de Pós-graduação, objetivando a sua revitalização, pois o mesmo encontrava-se com o Curso de Mestrado temporariamente desativado para o ingresso de novos alunos (1980-1983). Esta foi uma decisão da Reitoria da UFSC, em decorrência da falta de professores

** Cilei Chaves Rhodus foi professora e diretora da Escola Anna Nery.

Texto Contexto Enferm, Florianópolis, 2009 Out-Dez; 18(4): 759-65. 
doutores permanentes, do reduzido número de publicações e, principalmente, devido ao conceito C emitido pela CAPES. ${ }^{13}$

Com o retorno da Professora Eloita, agora Doutora, se estabelece um novo marco na enfermagem catarinense. A Pós-graduação passa por transformações e começa a ser organizado e implementado um Curso de Especialização. Bases mais sólidas para a enfermagem são estabelecidas, inclusive a partir da utilização de marcos conceituais para a Especialização, Mestrado e futuramente o Doutorado.

O Curso de Mestrado passou a adquirir uma identidade própria, focalizando a fundamentação teórico-filosófica da enfermagem. Houve uma maior preocupação com os instrumentos de pesquisa, a utilização de métodos e o estudo das teorias de enfermagem, que já vinham sendo trabalhadas por Wanda de Aguiar Horta. ${ }^{13}$ Além disso, o Programa passou a conviver com o processo de reestruturação permanente da produção acadêmica, adequando-o ao trabalho científico, realizado por docentes e discentes. Este trabalho, que anteriormente vinha se desenvolvendo a partir de pesquisas individuais, passa a ser desenvolvido em núcleos e a se inserir em áreas temáticas que constituem as linhas de investigação. Estas linhas passaram a se associar em grupos de pesquisa. ${ }^{11}$

De 1982 a 1991, além do intenso trabalho desenvolvido na Pós-graduação, a Professora Eloita, juntamente com a Professora Vera Radünz, desenvolveu um Projeto de Extensão no Centro de Pesquisas Oncológicas de Florianópolis, prestando cuidado a pessoas com câncer. Para este projeto estabeleceu bases teórico-práticas para a assistência de enfermagem fundamentada na Teoria de Imogene King.

Ainda nesse período, a Professora Eloita além de reorganizar o Curso de Pós-graduação em parceria com outras professoras, ministrou algumas disciplinas e orientou inúmeros alunos do Curso de Mestrado. Posteriormente, passou a preparar-se para cursar o Pós-Doutorado. Ingressa na University of Califórnia em São Francisco, Estados Unidos, em 1987, realizando estudos em enfermagem oncológica, desenvolvimento de conceitos e teorias e pesquisa qualitativa, permanecendo nos Estados Unidos até setembro de 1988. Retorna ao Brasil e continua ministrando disciplinas e orientando no Programa de Pós-graduação em Enfermagem até 1991, quando então se aposenta pela UFSC.
Mesmo como professora aposentada, continuou como colaboradora no Programa de Pósgraduação e criou em 1993, o Grupo de Pesquisa Cuidando e Confortando, vinculado ao PEN/ UFSC, que tem por objetivo desenvolver estudos e pesquisas na área do cuidado e do conforto ao ser humano. ${ }^{* * *}$ Desde sua criação, o Grupo vem sendo responsável por inúmeras pesquisas sobre a temática, das quais tem resultado produções científicas variadas, tais como: artigos, capítulos de livro, trabalhos apresentados em congressos nacionais e internacionais, clipes, entre outros. $\mathrm{O}$ Grupo também organizou importantes eventos, dentre eles, o $1^{\circ}$ Encontro Brasileiro de Cuidado e Conforto em Enfermagem, realizado em Itapema-SC, em 1996, que culminou em uma significativa produção científica publicada na Texto \& Contexto Enfermagem.

A partir do seu desligamento da UFSC, trabalhou como professora visitante na Escola de Enfermagem da Universidade Federal do Paraná (UFPR), de dezembro de 1992 a dezembro de 1996, sendo que devido ao convênio entre UFPR e UFSC pela Rede de Pós-graduação em Enfermagem da Região Sul, continuou atuando nos cursos de mestrado e doutorado da UFSC. Trabalhou também na Universidade Estadual do Rio de Janeiro, no período de 2000 a $2004 .^{12}$ Atualmente participa do Projeto Amanhecer, na área de Terapia Energética no Hospital Universitário, destinado a comunidade universitária, utilizandose de terapias naturais.

\section{O reconhecimento pela trajetória acadêmica e profissional}

A trajetória acadêmica e profissional da Professora Dra. Eloita Pereira Neves, juntamente com sua atuação frente à associação de classe e na construção e consolidação do Departamento e Curso de Graduação em Enfermagem da UFSC e dos Programas de Pós-graduação da Enfermagem catarinense e brasileira, merecem sem dúvida o reconhecimento institucional, nacional e internacional. Em 1996 foi agraciada, com medalha de mérito da Universidad Nacional de Altiplano - Puno, Peru. No ano de 1998, recebeu diploma de honra ao mérito do Colégio de Enfermería de Carabobo, na Venezuela. Em 2002, recebeu o certificado de reconhecimento assinado pela Coordenadora Internacional do Projeto das Escolas de

\footnotetext{
**** Programa de Pós-Graduação em Enfermagem. Folder do Grupo de Pesquisa Cuidando e ConfortandoC\&C; 2006.
} 
Enfermagem na área da Redução da Demanda na América Latina, Comissão Interamericana para o Controle de Abuso de Drogas e Organização dos Estados Americanos. Em 2003 recebeu da Escola de Enfermagem Anna Nery o prêmio - A Enfermagem e a Saúde do trabalhador. Recentemente foi homenageada pela Escola de Enfermagem da Universidade Federal do Estado do Rio de Janeiro, quando teve seu nome registrado em um dos prêmios de importante evento científico realizado por essa instituição. ${ }^{12}$ No ano em curso, foi homenageada especial do Departamento/ Curso de Graduação em Enfermagem nas atividades de comemoração dos 40 anos de sua criação. Em junho de 2009 foi agraciada com o Título de "Professora Emérita" outorgado pela Universidade Federal de Santa Catarina, sendo a primeira enfermeira docente e a sexta mulher homenageada com esta honraria nos 50 anos de existência da UFSC, e em outubro do mesmo ano recebeu em Sessão Especial, Comenda oferecida pelo Conselho Federal de Enfermagem.

\section{CONCLUSÃO}

Os resultados deste estudo demonstram o valor expressivo da Professora Eloita e a sua contribuição efetiva para o fortalecimento da profissão e do ensino da Enfermagem. Por seu espírito arrojado, comprometimento pessoal e profissional, a professora empreendeu uma trajetória de sucesso reconhecida pela Enfermagem catarinense e brasileira. A sua dedicação ao ensino, pesquisa e extensão na área da Enfermagem merece, sem dúvida, o devido reconhecimento do Departamento/Curso de Graduação em Enfermagem na comemoração dos seus 40 anos de existência.

\section{REFERÊNCIAS}

1. Baptista SS, Barreira IA. Enfermagem de nível superior no Brasil e vida associativa. Rev Bras Enferm. 2006; 59(Esp):411-6.
2. Sauthier, J. A missão das enfermeiras norte americanas na capital da República (1921-1931) [tese]. Rio de Janeiro (RJ): Escola de Enfermagem Anna Nery. Universidade Federal do Rio de Janeiro; 1998.

3. Borenstein MS, AlthoffCR. Projetandoe conquistando um caminho para a formação profissional do enfermeiro. In: Boresntein MS, Althoff CR, Souza ML. Enfermagem da UFSC: recortes de caminhos construídos e memórias (1969-1999). Florianópolis (SC): Insular; 1999. p. 25-64.

4. Bardin, L. Análise de conteúdo. Lisboa (PT). Edições 70; 2004.

5. Borenstein MS. Antecedentes históricos, sua criação e a primeira turma de formandos. [Monografia apresentada para concurso Professor Adjunto] Florianópolis (SC): Universidade Federal de Santa Catarina; 1994.

6. Carvalho AC. Associação Brasileira de Enfermagem 1926-1986. Documentário. Brasília (DF): ABEn; 1976.

7. Associação Brasileira de Enfermagem - Seção Santa Catarina. Gestão das Diretorias. Banner Florianópolis (SC); 2001.

8. Mendes NTC. A contribuição da ABEn-SC nos 75 anos da ABEn. Rev Bras Enferm. 2006 Abr-Jun; 54(2):356-63.

9. Vale Pereira N. Associação Irmão Joaquim, 100 anos de amor ao próximo: histórico da associação Irmão Joaquim por ocasião do seu centenário 1902-2002. Nereu do Vale Pereira. Florianópolis (SC): Associação Irmão Joaquim, 2002.

10. Rocha SMM, Almeida MCP, Wright MGM, Vieira, TT. O ensino de Pós-graduação no Brasil. São Paulo (SP): Cortez Editora; 1989.

11. Padilha MICS, Borenstein, MS, Maia, AR, Guedes, JAD, Lessmann JC, Machado C. Uma história de sucesso: 30 anos da Pós Graduação em Enfermagem da UFSC. Texto Contexto Enferm. 2006; 15(Esp):20-30.

12. Currículo Lattes, Eloita Pereira Neves. [página na internet]. [acesso 2008 Nov 11]. Disponível em http: // buscatextual.cnpq.Br/buscatextual/visualisacv. jsp?id=K4781744D1

13. Althoff CR, Borenstein, MS, Lunardi, VL, Heck, R. Conhecendo o conhecimento das dissertações de mestrado em enfermagem da UFSC. Texto Contexto Enferm. 1996, 5 (Esp):215-37. 